\title{
IDIOPATHIC INTRACRANIAL HYPERTENSION AFTER HYSTEROSCOPIC EVALUATION OF ABNORMAL UTERINE BLEEDING
}

\author{
Zahra Asgari1 ${ }^{1}$ Fetemeh Tabatabaei², Reihaneh Hosseini3 ${ }^{3}$, Zahra Tavoli ${ }^{4}$
}

${ }_{1}^{1}$ Associate Professor, Department of Obstetrics and Gynaecology, Arash Women's Hospital, Tehran University of Medical Sciences, Tehran, Iran.

${ }^{2}$ Assistant Professor, Department of Obstetrics and Gynaecology, Shahid Mahallati Hospital, Tabriz University of Medical Sciences, Tabriz, Iran.

${ }^{3}$ Assistant Professor, Department of Obstetrics and Gynaecology, Arash Women's Hospital, Tehran University of Medical Sciences, Tehran, Iran.

${ }^{4}$ Assistant Professor, Department of Obstetrics and Gynaecology, Ziaeian Hospital, Tehran University of Medical Sciences, Tehran, Iran.

HOW TO CITE THIS ARTICLE: Asgari Z, Tabatabaei F, Hosseini R, et al. Idiopathic intracranial hypertension after hysteroscopic evaluation of abnormal uterine bleeding. J. Evolution Med. Dent. Sci. 2019;8(03):223-226, DOI: 10.14260/jemds/2019/48

\begin{abstract}
PRESENTATION OF CASE
Abnormal Uterine Bleeding (AUB), is a common gynaecologic problem and affecting approximately 10 to 35 percent of women.(1) Polycystic ovary syndrome (PCOS) is a common used as the distending medium with the appropriate pressure of rotatory pump. The procedure had been terminated at 1520 minutes after polyp resection. There was no other complication during the operation.
\end{abstract} endocrine disorder affecting women in the reproductive age. It is associated with the obesity, metabolic disorders, hypertension and abnormal uterine bleeding.(2) Hysteroscopy provides a minimally invasive approach to abnormal uterine bleeding and also has the potential benefit of combining evaluation with the treatment.

Idiopathic intracranial hypertension (IIH), which is commonly called pseudotumor cerebri, is a disorder characterized by symptoms and signs of higher intracranial pressure such as headache, papilledema and vision loss without any other cause of intracranial hypertension on neuroimaging and with normal cerebrospinal fluid composition.(3) The incidence of IIH is 1 to 2 per 100, 000 populations per year.(4) IIH primarily affects overweight women at the childbearing age. A great number of systemic diseases (Polycystic ovary syndrome), drugs (growth hormone, tetracyclines, and retinoids), vitamin deficiency and excesses (Hypervitaminosis A), recent weight gain and hereditary conditions probably increase the incidence of IIH.

A 36-year-old lady with $\mathrm{G}_{3} \mathrm{P}_{2}$ (NVD) $\mathrm{EP}_{1}$ referred to an outpatient gynaecology clinic with severe headache, nausea, intermittent vomiting and subsequent lower visual acuity for two weeks, initiated a few hours after hysteroscopic evaluation of uterine cavity due to the abnormal uterine bleeding. She suffered from intermittent periods of oligomenorrhea with subsequent polymenorrhoea for more than a year with recently worsened menorrhagia. Increased endometrial thickness was found on an ultrasound study on the uterine cavity with the probability of endometrial polyp.

She had been admitted for hysteroscopic evaluation of endometrial cavity two weeks ago and glycine $1.5 \%$ had been

\section{DIFFERENTIAL DIAGNOSIS}

1. Hysteroscopy medium related electrolyte imbalance.

2. Obstruction of venous outflow, e.g., venous sinus thrombosis.

3. Intracranial mass lesions (Tumour, Abscess).

4. Malignant systemic hypertension.

\section{PATHOLOGICAL DISCUSSION}

The primary laboratory study was normal. Body weight was $90 \mathrm{Kg}$ and the body mass index (BMI) was $33 \mathrm{Kg} / \mathrm{m}^{2}$. There was no previous history of any recent weight gain, hypertension, headache, visual disturbance, drug administration or oral contraceptives use in her past history. She was hospitalized immediately for completing the neurologic and ophthalmologic evaluation. The brain magnetic resonance imaging and venography studies were normal. The cerebrospinal fluid pressure was more than 50 $\mathrm{cm} \mathrm{H}_{2} \mathrm{O}$ with the $\mathrm{Hb}$ value of $8.2 \mathrm{~g} / \mathrm{dl}$. Other laboratory examinations were also normal. Ophthalmologic studies showed bilateral visual field defects (Fig. 1). Acetazolamide administration in addition to therapeutic lumbar puncture, blood transfusion and other conservative therapies were done, and she was finally discharged with a partial visual impairment. Further evaluations exhibited a visual impairment, which lasted for other six months, in addition to irregular menstrual cycles (Fig. 2).

'Financial or Other Competing Interest': None.

Submission 15-12-2018, Peer Review 08-01-2019,

Acceptance 14-01-2019, Published 21-01-2019.

Corresponding Author:

Fatemeh Tabatabaei,

Assistant Professor,

Shahid Mahallati Hospital,

Tabriz University of Medical Sciences,

Tabriz, Iran.

E-mail:drtabatabaeigyn@gmail.com

DOI: $10.14260 / \mathrm{jemds} / 2019 / 48$

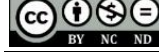




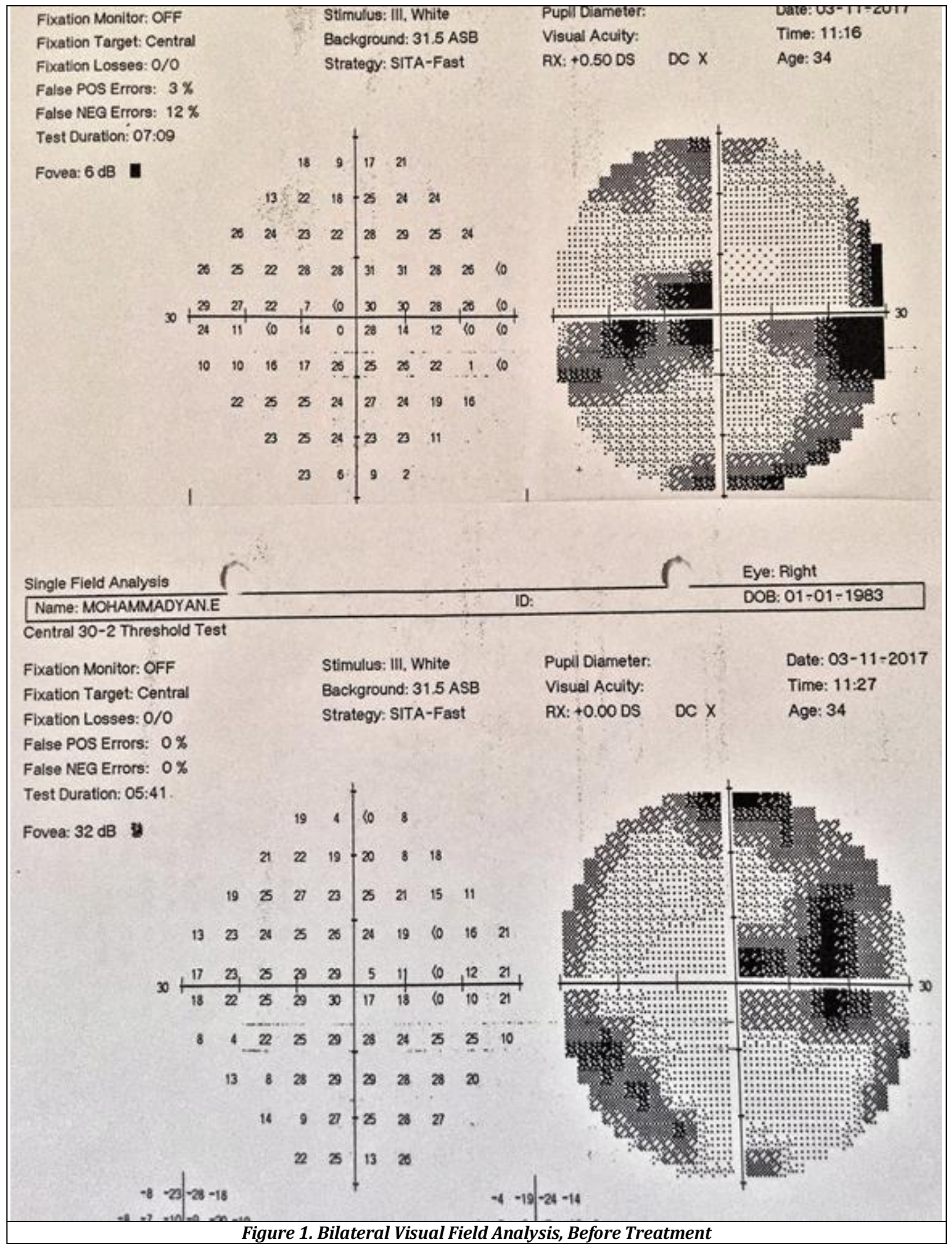



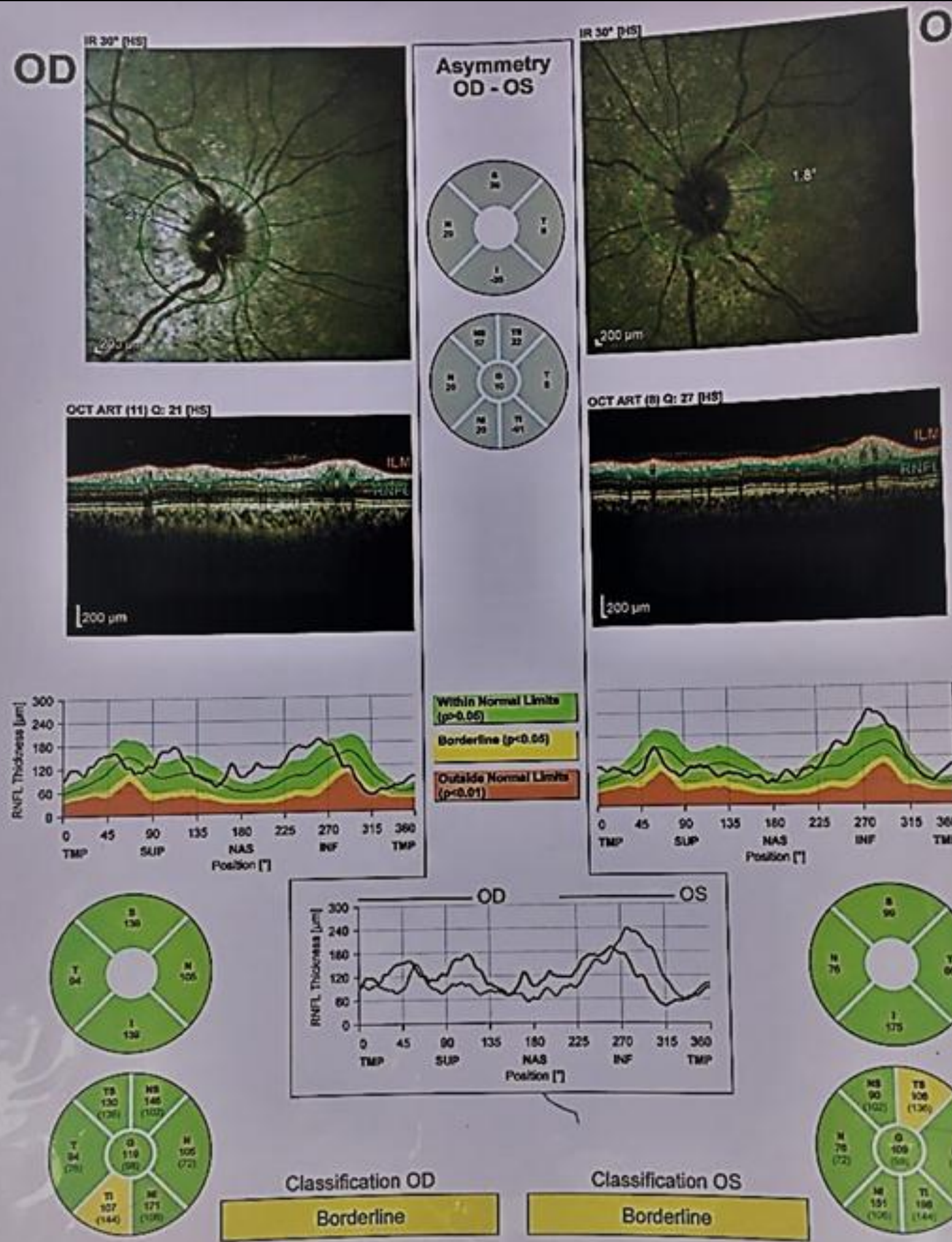

Classification OD

Borderline
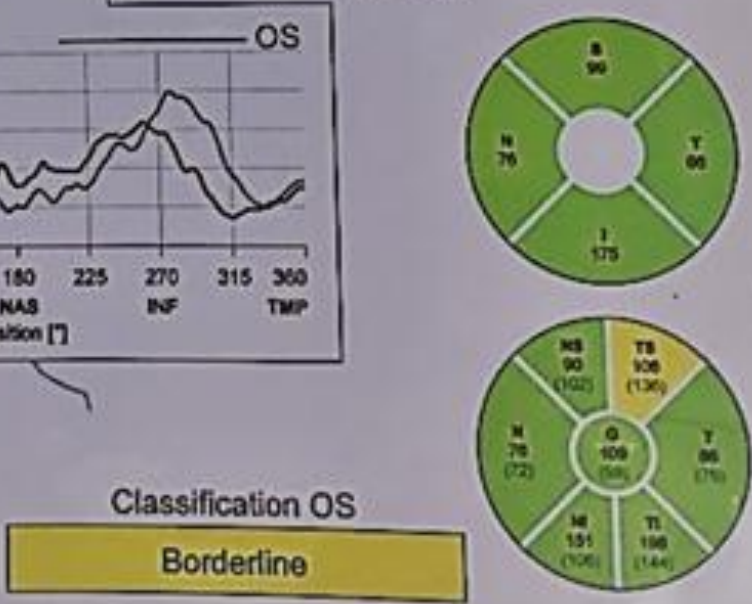

Figure 2. Bilateral Visual Field Analysis, After Treatment

\section{DISCUSSION OF MANAGEMENT}

There is no certain evidence for increased intracranial hypertension and reduced visual acuity related to the hysteroscopy and distending media or the existence of two concurrent phenomena. Fluid overload causes hypervolemia and consequent dilutional hyponatremia. The resultant cerebral oedema may cause the agitation, confusion, weakness, visual disturbances, blindness and headache.(5,6) A distending medium related electrolyte imbalance and individual susceptibility may play roles in this field. In conclusion, patients with the PCOS are susceptible to the IIH.(7) Despite the gradual improvement development by the treatment, symptoms may be slowly worsened leading to the persistent papilledema, elevated intracranial pressure and permanent visual loss. ${ }^{(8)}$ Therefore, there is a need for more attention to fluid deficits and electrolyte balance in hysteroscopic evaluations of AUB in patients with PCOS. 


\section{FINAL DIAGNOSIS}

Idiopathic Intracranial Hypertension.

\section{Abbreviations}

AUB- Abnormal Uterine Bleeding.

PCOS- Polycystic Ovary Syndrome.

IIH- Idiopathic Intracranial Hypertension.

NVD- Normal Vaginal Delivery.

BMI- Body Mass Index.

\section{REFERENCES}

[1] Cote I, Jacobs P, Cumming DC. Use of health services associated with increased menstrual loss in the United States. American Journal of Obstetrics and Gynecology 2003;188(2):343-8.

[2] Bentley-Lewis R, Seely E, Dunaif A. Ovarian hypertension: polycystic ovary syndrome. Endocrinol Metab Clin North Am 2011;40(2):433-49.

[3] Friedman DI, Jacobson DM. Diagnostic criteria for idiopathic intracranial hypertension. Neurology 2002;59(10):1492-5.
[4] Durcan FJ, Corbett JJ, Wall M. The incidence of pseudotumor cerebri: population studies in Iowa and Louisiana. Archives of Neurology 1988;45(8):875-7.

[5] Umranikar S, Clark TJ, Saridogan E, et al. BSGE/ESGE guideline on management of fluid distension media in operative hysteroscopy. Gynecol Surg 2016;13(4):289-303.

[6] Istre 0, Bjoennes J, Naess R, et al. Postoperative cerebral oedema after transcervical endometrial resection and uterine irrigation with $1.5 \%$ glycine. The Lancet 1994;344(8931):1187-9.

[7] Avisar I, Gaton DD, Dania H, et al. The prevalence of polycystic ovary syndrome in women with idiopathic intracranial hypertension. Scientifica (Cairo) 2012;(2012):708042.

[8] Best J, Silvestri G, Burton B, et al. The incidence of blindness due to idiopathic intracranial hypertension in the UK. Open Ophthalmol Journal 2013;7:26-9. 